\title{
A TRANSMISSION PROBLEM FOR BEAMS ON NONLINEAR SUPPORTS
}

\author{
TO FU MA AND HIGIDIO PORTILLO OQUENDO
}

Received 20 October 2005; Revised 10 April 2006; Accepted 12 April 2006

A transmission problem involving two Euler-Bernoulli equations modeling the vibrations of a composite beam is studied. Assuming that the beam is clamped at one extremity, and resting on an elastic bearing at the other extremity, the existence of a unique global solution and decay rates of the energy are obtained by adding just one damping device at the end containing the bearing mechanism.

Copyright (C) 2006 T. F. Ma and H. Portillo Oquendo. This is an open access article distributed under the Creative Commons Attribution License, which permits unrestricted use, distribution, and reproduction in any medium, provided the original work is properly cited.

\section{Introduction}

In this paper we consider the existence of a global solution and decay rates of the energy for a transmission problem involving two Euler-Bernoulli equations with nonlinear boundary conditions. More precisely, we are concerned with the system of equations

$$
\begin{array}{ll}
\rho_{1} u_{t t}+\beta_{1} u_{x x x x}=0 & \text { in }] 0, L_{0}\left[\times \mathbb{R}^{+},\right. \\
\rho_{2} v_{t t}+\beta_{2} v_{x x x x}=0 & \text { in }] L_{0}, L\left[\times \mathbb{R}^{+},\right.
\end{array}
$$

coupled by the "transmission" conditions

$$
\begin{aligned}
u\left(L_{0}, t\right)-v\left(L_{0}, t\right)=0, & u_{x}\left(L_{0}, t\right)-v_{x}\left(L_{0}, t\right)=0, \\
\beta_{1} u_{x x}\left(L_{0}, t\right)-\beta_{2} v_{x x}\left(L_{0}, t\right)=0, & \beta_{1} u_{x x x}\left(L_{0}, t\right)-\beta_{2} v_{x x x}\left(L_{0}, t\right)=0 .
\end{aligned}
$$

To the system we add the nonlinear boundary conditions

$$
\begin{gathered}
u(0, t)=0, \quad u_{x}(0, t)=0, \\
v_{x x}(L, t)=0, \quad \beta_{2} v_{x x x}(L, t)=f(v(L, t))+g\left(v_{t}(L, t)\right),
\end{gathered}
$$




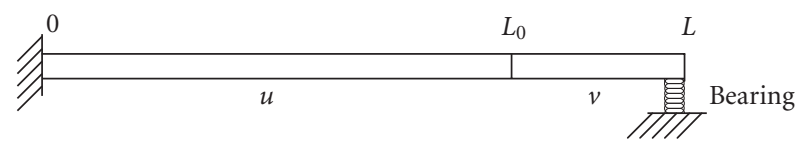

Figure 1.1. A composite beam on an elastic bearing.

and the initial data

$$
\begin{aligned}
& u(x, 0)=u^{0}(x), \quad u_{t}(x, 0)=u^{1}(x) \text { in }\left[0, L_{0}\right], \\
& v(x, 0)=v^{0}(x), \quad v_{t}(x, 0)=v^{1}(x) \quad \text { in }\left[L_{0}, L\right] .
\end{aligned}
$$

The system (1.1)-(1.6) models the transverse vibrations of a composite beam of length $L$, constituted by two types of materials of different mass densities $\rho_{1}, \rho_{2}>0$ and flexural rigidities $\beta_{1}, \beta_{2}>0$. Because of the boundary condition (1.4), the beam is clamped at the left end $x=0$. On the other extremity, the condition (1.5) implies that the bending moment is zero and that the shear force is equal to $f(v(L, t))+g\left(v_{t}(L, t)\right)$. This means that, at the end $x=L$, the beam is resting on a kind of bearing, described by the function $f$, and subjected to a frictional dissipation described by the function $g$ (see Figure 1.1).

We notice that stabilization of transmission problems has been considered by some authors. In the beginning, Lions [8] studied the exact controllability of the transmission problem for the wave equation. Later, Liu and Williams [10] studied the boundary stabilization of transmission problems for linear systems of wave equations. In the case of beam equations, which involve fourth-order derivatives, there are more possibilities in the problem modeling and boundary conditions. For instance, Muñoz Rivera and Portillo Oquendo [14] studied a transmission problem for viscoelastic beams, by exploiting the dissipations due to the memory effects of the material. On the other hand, there are a few results on fourth-order equations with nonlinear boundary conditions involving third-order derivatives. That class of problems models elastic beams on elastic bearings, and one of the first results, with nonlinearities, was given by Feireisl [2], who studied the periodic solutions for a superlinear problem. Some related stationary problems were considered by Grossinho and Ma [3] and Ma [11]. We refer the reader to [1, 4-7, 12-16] for other interesting related works.

Our objective is to show that under suitable assumptions, the sole dissipation $g\left(v_{t}\right)$, acting on the boundary point $x=L$, will be sufficient to stabilize the whole system. The dissipation effect on the boundary $x=L$ will be transmitted to (1.1) through (1.2). The proof of the boundary stabilization is based on the arguments from Lagnese [6] and Lagnese and Leugering [7].

The paper is organized as follows. In Section 2 we define some notations and establish the global existence and uniqueness results (see Theorem 2.2). Weak solutions are also considered (see Theorem 2.6). In Section 3 we prove the decay of the energy of the system, 
which is defined by

$$
\begin{aligned}
E(t)=E(t, u, v)= & \frac{1}{2} \int_{0}^{L_{0}}\left\{\rho_{1}\left|u_{t}\right|+\beta_{1}\left|u_{x x}\right|\right\} d x \\
& +\frac{1}{2} \int_{L_{0}}^{L}\left\{\rho_{2}\left|v_{t}\right|+\beta_{2}\left|v_{x x}\right|\right\} d x+\hat{f}(v(L, t)),
\end{aligned}
$$

where $\hat{f}(w)=\int_{0}^{w} f(s) d s$ (see Theorem 3.1).

\section{Global existence}

In our study we assume that $f$ is a $C^{1}$ function satisfying the sign condition

$$
f(w) w \geq 0, \quad \forall w \in \mathbb{R},
$$

and that $g$ is a $C^{1}$ for which there exists a constant $c_{0}>0$ such that

$$
g(0)=0, \quad(g(r)-g(s))(r-s) \geq c_{0}|r-s|^{2}, \quad \forall r, s \in \mathbb{R} .
$$

In particular it follows that $g(w) w \geq c_{0} w^{2}$ for all $w \in \mathbb{R}$. In order to deal with the transmission conditions (1.3) and the boundary condition (1.4), we define the Sobolev space

$$
X=\left\{(\varphi, \psi) \in \mathbb{T}^{2} \mid(\varphi, \psi) \text { satisfies }(2.4)\right\},
$$

where

$$
\begin{gathered}
\varphi(0)=\varphi_{x}(0)=\varphi\left(L_{0}\right)-\psi\left(L_{0}\right)=\varphi_{x}\left(L_{0}\right)-\psi_{x}\left(L_{0}\right)=0, \\
\mathbb{T}^{k}=H^{k}\left(0, L_{0}\right) \times H^{k}\left(L_{0}, L\right) .
\end{gathered}
$$

We also write $\mathbb{L}^{2}=L^{2}\left(0, L_{0}\right) \times L^{2}\left(L_{0}, L\right)$. Our study is based on the space

$$
V=\left\{(\varphi, \psi) \in\left(H^{2}\left(0, L_{0}\right) \times H^{3}\left(L_{0}, L\right)\right) \cap X \mid \psi_{x x}(L)=0\right\},
$$

so that the first part of condition (1.5) is also recovered. As a simple consequence of the trace theorem and (2.4) one has the following useful boundary estimate.

Lemma 2.1. Given $(u, v) \in C^{1}([0, T], X)$, there exists a constant $C>0$ such that

$$
\begin{array}{ll}
|v(L, t)| \leq C\left\{\left\|u_{x x}\right\|_{2}+\left\|v_{x x}\right\|_{2}\right\}, & \forall t \in[0, T], \\
\left|v_{t}(L, t)\right| \leq C\left\{\left\|u_{x x t}\right\|_{2}+\left\|v_{x x t}\right\|_{2}\right\}, & \forall t \in[0, T],
\end{array}
$$

where $\|\cdot\|_{2}$ denotes either $L^{2}\left(0, L_{0}\right)$ or $L^{2}\left(L_{0}, L\right)$ norms.

Now we prove the existence of global regular solutions. 
4 A transmission problem for beams on nonlinear supports

Theorem 2.2. Assume that conditions (2.1)-(2.2) hold. Then for any initial data $\left(u^{0}, v^{0}\right) \in$ $\mathbb{\square}^{4} \cap V$ and $\left(u^{1}, v^{1}\right) \in V$, satisfying the compatibility condition,

$$
\begin{gathered}
v_{x x x}^{0}(L)-f\left(v^{0}(L)\right)-g\left(v^{1}(L)\right)=0, \\
\beta_{1} u_{x x}^{0}\left(L_{0}, t\right)-\beta_{2} v_{x x}^{0}\left(L_{0}, t\right)=0, \\
\beta_{1} u_{x x x}^{0}\left(L_{0}, t\right)-\beta_{2} v_{x x x}^{0}\left(L_{0}, t\right)=0,
\end{gathered}
$$

problem (1.1)-(1.6) has a unique strong solution $(u, v)$ such that

$$
(u, v) \in L^{\infty}\left(\mathbb{R}^{+} ; \mathbb{W}^{4}\right), \quad\left(u_{t}, v_{t}\right) \in L^{\infty}\left(\mathbb{R}^{+} ; X\right), \quad\left(u_{t t}, v_{t t}\right) \in L^{\infty}\left(\mathbb{R}^{+} ; \mathbb{L}^{2}\right) .
$$

The proof of Theorem 2.2 is given in several steps, by using the Galerkin method.

Approximate problem. Let $\left\{\left(\varphi^{n}, \psi^{n}\right)\right\}_{n \in \mathbb{N}}$ be a Galerkin basis of $V$, which for convenience is chosen to satisfy

$$
\left\{\left(u^{0}, v^{0}\right),\left(u^{1}, v^{1}\right)\right\} \subset V_{2},
$$

where

$$
V_{m}=\operatorname{span}\left\{\left(\varphi^{1}, \psi^{1}\right), \ldots,\left(\varphi^{m}, \psi^{m}\right)\right\}
$$

Then the corresponding approximate variational problem to problem (1.1)-(1.6) reads as follows: find $\left(u^{m}(t), v^{m}(t)\right) \in V_{m}$ of the form

$$
\left(u^{m}(t), v^{m}(t)\right)=\sum_{j=1}^{m} h_{j}^{m}(t)\left(\varphi^{j}, \psi^{j}\right)
$$

such that

$$
\begin{aligned}
& \int_{0}^{L_{0}}\left\{\rho_{1} u_{t t}^{m} \varphi^{j}+\beta_{1} u_{x x}^{m} \varphi_{x x}^{j}\right\} d x+\int_{L_{0}}^{L}\left\{\rho_{2} v_{t t}^{m} \psi^{j}+\beta_{2} v_{x x}^{m} \psi_{x x}^{j}\right\} d x \\
& \quad+\left\{\beta_{2} f\left(v^{m}(L, t)\right)+g\left(v_{t}^{m}(L, t)\right)\right\} \psi^{j}(L)=0, \\
& \left(u^{m}(0), v^{m}(0)\right)=\left(u^{0}, v^{0}\right), \quad\left(u_{t}^{m}(0), v_{t}^{m}(0)\right)=\left(u^{1}, v^{1}\right) .
\end{aligned}
$$

As a matter of fact, $(2.13)$ is an $m$-dimensional system of ODEs in $h_{j}^{m}(t)$ and has a local solution $\left(u^{m}(t), v^{m}(t)\right)$ in an interval $\left[0, t_{m}\right]$. In the following, we derive uniform estimates, so that local solutions can be extended to the interval $[0, T]$ for any $T>0$. Note that initial conditions in (2.14) are well defined because of (2.10).

Estimate 2.3. Replacing $\varphi^{i}$ by $u_{t}^{m}$ and $\psi^{i}$ by $v_{t}^{m}$ in (2.13), one concludes that

$$
\frac{d}{d t} E\left(t, u^{m}, v^{m}\right)=-\beta_{2} g\left(v_{t}^{m}(L, t)\right) v_{t}^{m}(L, t)
$$


Then from condition (2.2) we see that $E\left(t, u^{m}, v^{m}\right)$ is decreasing and therefore there exists $M_{1}>0$ such that

$$
\left\|u_{t}^{m}(t)\right\|_{2}^{2}+\left\|v_{t}^{m}(t)\right\|_{2}^{2}+\left\|u_{x x}^{m}(t)\right\|_{2}^{2}+\left\|v_{x x}^{m}(t)\right\|_{2}^{2} \leq M_{1}
$$

for all $m \in \mathbb{N}, t>0$, where $M_{1}$ depends on $E\left(0, u^{0}, v^{0}\right)$.

Estimate 2.4. Let us obtain an estimate for $u_{t t}^{m}(0)$ and $v_{t t}^{m}(0)$ in $L^{2}$ norms. Replacing $\varphi^{i}$ by $u_{t t}^{m}(0)$ and $\psi^{i}$ by $v_{t t}^{m}(0)$ in (2.13), one concludes from the compatibility condition (2.8) that for some constant $C>0$,

$$
\left\|u_{t t}^{m}(0)\right\|_{2}^{2}+\left\|v_{t t}^{m}(0)\right\|_{2}^{2} \leq C\left(\left\|u_{x x x x}^{0}\right\|_{2}^{2}+\left\|v_{x x x x}^{0}\right\|_{2}^{2}\right)
$$

Therefore, there exists $M=M\left(u^{0}, v^{0}\right)>0$ such that

$$
\left\|u_{t t}^{m}(0)\right\|_{2}^{2}+\left\|v_{t t}^{m}(0)\right\|_{2}^{2} \leq M
$$

for all $m \in \mathbb{N}$.

Estimate 2.5. Here we use a finite-difference argument as in [12]. Let us fix $t, \xi>0$ such that $\xi<T-t$, and take the difference of (2.13) with $t=t+\xi$ and $t=t$. Then replacing $\varphi^{j}$ by $u_{t}^{m}(t+\xi)-u_{t}^{m}(t)$ and $\psi^{j}$ by $v_{t}^{m}(t+\xi)-v_{t}^{m}(t)$, and putting

$$
\begin{aligned}
\hat{P}_{m}(t, \xi)= & \rho_{1}\left\|u_{t}^{m}(t+\xi)-u_{t}^{m}(t)\right\|_{2}^{2}+\rho_{2}\left\|v_{t}^{m}(t+\xi)-v_{t}^{m}(t)\right\|_{2}^{2} \\
& +\beta_{1}\left\|u_{x x}^{m}(t+\xi)-u_{x x}^{m}(t)\right\|_{2}^{2}+\beta_{2}\left\|v_{x x}^{m}(t+\xi)-v_{x x}^{m}(t)\right\|_{2}^{2},
\end{aligned}
$$

one infers that

$$
\frac{1}{2} \frac{d}{d t} \widehat{P}_{m}(t, \xi) \leq A+B
$$

where

$$
\begin{gathered}
A=-\left(g\left(v^{m}(L, t+\xi)\right)-g\left(v^{m}(L, t)\right)\right)\left(v_{t}^{m}(L, t+\xi)-v_{t}^{m}(L, t)\right), \\
B=-\beta_{2}\left(f\left(v^{m}(L, t+\xi)\right)-f\left(v^{m}(L, t)\right)\right)\left(v_{t}^{m}(L, t+\xi)-v_{t}^{m}(L, t)\right) .
\end{gathered}
$$

Taking $0<\varepsilon<c_{0}$, and using the mean value theorem and Lemma 2.1, there exists $C_{\varepsilon}>0$ such that

$$
\begin{aligned}
B \leq & C_{\varepsilon}\left(\left\|u_{x x}^{m}(t+\xi)-u_{x x}^{m}(t)\right\|_{2}^{2}+\left\|v_{x x}^{m}(t+\xi)-v_{x x}^{m}(t)\right\|_{2}^{2}\right) \\
& +\varepsilon\left|v_{t}^{m}(L, t+\xi)-v_{t}^{m}(L, t)\right|^{2} .
\end{aligned}
$$

Then from condition (2.2), we conclude that for a constant $C>0$,

$$
\frac{1}{2} \frac{d}{d t} \widehat{P}_{m}(t, \xi)=C \hat{P}_{m}(t, \xi)
$$


6 A transmission problem for beams on nonlinear supports

and therefore $\hat{P}_{m}(t, \xi) \leq \hat{P}_{m}(0, \xi) e^{2 C T}$. So, dividing the inequality by $\xi^{2}$ and making $\xi \rightarrow 0$, we see that

$$
\begin{aligned}
& \rho_{1}\left\|u_{t t}^{m}(t)\right\|_{2}^{2}+\rho_{2}\left\|v_{t t}^{m}(t)\right\|_{2}^{2}+\beta_{1}\left\|u_{x x t}^{m}(t)\right\|_{2}^{2}+\beta_{2}\left\|v_{x x t}^{m}(t)\right\|_{2}^{2} \\
& \quad \leq\left(\rho_{1}\left\|u_{t t}^{m}(0)\right\|_{2}^{2}+\rho_{2}\left\|v_{t t}^{m}(0)\right\|_{2}^{2}+\beta_{1}\left\|u_{x x}^{1}\right\|_{2}^{2}+\beta_{2}\left\|v_{x x}^{1}\right\|_{2}^{2}\right) e^{C T} .
\end{aligned}
$$

Hence there exists $M_{2}>0$ such that

$$
\left\|u_{t t}^{m}(t)\right\|_{2}^{2}+\left\|v_{t t}^{m}(t)\right\|_{2}^{2}+\left\|u_{x x t}^{m}(t)\right\|_{2}^{2}+\left\|v_{x x t}^{m}(t)\right\|_{2}^{2} \leq M_{2}
$$

for all $m \in \mathbb{N}$ and $t \in[0, T]$.

Existence result. From Estimates 2.3 and 2.5, we can apply Aubin-Lions compactness theorem to pass to the limit the approximate problem. Then the proof of the existence result is complete.

Uniqueness. Let $\left(u_{1}, v_{1}\right)$ and $\left(u_{2}, v_{2}\right)$ be two solutions of problem (1.1)-(1.6). Writing $U=u_{1}-u_{2}$ and $V=v_{1}-v_{2}$, we see that $(U, V)$ satisfies

$$
\begin{gathered}
\frac{1}{2} \frac{d}{d t}\left\{\rho_{1}\left\|U_{t}(t)\right\|_{2}^{2}+\rho_{2}\left\|V_{t}(t)\right\|_{2}^{2}+\beta_{1}\left\|U_{x x}(t)\right\|_{2}^{2}+\beta_{2}\left\|V_{x x}(t)\right\|_{2}^{2}\right\} \\
\leq-\beta_{2}\left[f\left(v_{1}(L, t)\right)-f\left(v_{2}(L, t)\right)\right] V_{t}(L, t) \\
-\beta_{2}\left[g\left(v_{1 t}(L, t)\right)-g\left(v_{2 t}(L, t)\right)\right] V_{t}(L, t) .
\end{gathered}
$$

Then using (2.2) and Lemma 2.1, as in Estimate 2.5, we deduce the existence of $C>0$ such that

$$
\frac{d}{d t} P(t) \leq C\left(\left\|U_{x x}(t)\right\|_{2}^{2}+\left\|V_{x x}(t)\right\|_{2}^{2}\right), \quad t \in[0, T]
$$

where now $P(t)=P(U, V, t)$. Since we have $P(0)=0$, from Gronwall lemma we get $U=$ $V=0$.

Weak solutions. We say that a pair $(u, v)$ is a weak solution of problem (1.1)-(1.6) if

$$
(u, v) \in L^{\infty}\left(\mathbb{R}^{+}, X\right), \quad\left(u_{t}, v_{t}\right) \in L^{\infty}\left(\mathbb{R}^{+}, \mathbb{L}^{2}\right), \quad\left(u_{t t}, v_{t t}\right) \in L^{\infty}\left(\mathbb{R}^{+}, \mathbb{W}^{-2}\right)
$$

satisfy the initial conditions (1.6), the compatibility conditions (2.8), and the variational identity

$$
\begin{aligned}
& \frac{d}{d t}\left\{\int_{0}^{L_{0}} \rho_{1} u_{t} \varphi d x+\int_{L_{0}}^{L} \rho_{2} v_{t} \psi d x\right\} \\
& \quad+\int_{0}^{L_{0}} \beta_{1} u_{x x} \varphi_{x x} d x+\int_{L^{0}}^{L} \beta_{2} v_{x x} \psi_{x x} d x+\left\{\beta_{2} f(v(L, t))+g\left(v_{t}(L, t)\right)\right\} \psi(L)=0
\end{aligned}
$$


for all $(\varphi, \psi) \in X$. In order to study the existence of weak solutions let us denote by $\mathscr{C}$ the set of all acceptable initial data for the existence of strong solutions, that is,

$$
\mathscr{C}:=\left\{\left(\left(u^{0}, v^{0}\right),\left(u^{1}, v^{1}\right)\right) \in\left(\mathbb{H}^{4} \cap V\right) \times V \mid(2.8) \text { holds }\right\} .
$$

Then we have the following existence result for weak solutions.

Theorem 2.6. Assume that conditions (2.1)-(2.2) hold. Then for any initial data satisfying

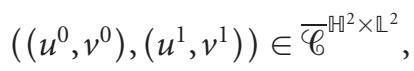

problem (1.1)-(1.6) has a unique weak solution.

This theorem is proved using density arguments, similar to those used by Cavalcanti et al. [1]. In fact, from the assumption on the initial data, there exists a sequence $\left(\left(u_{\nu}^{0}, v_{\nu}^{0}\right)\right.$, $\left.\left(u_{\nu}^{1}, v_{\nu}^{1}\right)\right) \in \mathscr{C}$ such that

$$
\left(u_{v}^{0}, v_{v}^{0}\right) \longrightarrow\left(u^{0}, v^{0}\right) \quad \text { in } \mathbb{T}^{2}, \quad\left(u_{v}^{1}, v_{v}^{1}\right) \longrightarrow\left(u^{1}, v^{1}\right) \quad \text { in } \mathbb{L}^{2} .
$$

Now, for each $v \in \mathbb{N}$, the initial conditions $\left(u_{\nu}^{0}, v_{v}^{0}\right)$ and $\left(u_{\nu}^{1}, v_{\nu}^{1}\right)$ give a unique regular solution $\left(u_{v}, v_{v}\right)$ of problem (1.1)-(1.6). From the estimates used in the proof of Theorem 2.2 it can be shown that $\left(u_{v}, v_{v}\right)$ converges to a weak solution $(u, v)$ of $(1.1)-(1.6)$. The uniqueness is then proved by means of the regularization techniques as by Lions and Visik (see e.g. [9]).

\section{Decay of the energy}

In this section we study decay rates for the first-order energy (1.7) associated to system (1.1)-(1.6). Here we assume that the bearing device has a superlinear behavior, characterized by the condition

$$
\exists \rho \geq 2 \text { such that } \rho \hat{f}(w)-f(w) w \leq 0, \forall w \in \mathbb{R},
$$

and that the material of the beam occupying $\left[L_{0}, L\right]$ is more dense and stiff than that in $\left[0, L_{0}\right]$, that is,

$$
\rho_{1} \leq \rho_{2}, \quad \beta_{1} \geq \beta_{2} .
$$

Then the rate of decay will depend on the behavior of the nonlinear dissipation $g$ in a neighborhood of the origin, which is related to the following assumption: there exist $c_{1}, c_{2}>0$ and $q \geq 1$ such that

$$
c_{1} \min \left\{|w|,|w|^{q}\right\} \leq|g(w)| \leq c_{2} \max \left\{|w|,|w|^{1 / q}\right\} .
$$

Our main result is given by the following theorem.

Theorem 3.1. Suppose that

$$
\left(u^{0}, v^{0}\right) \in \mathbb{H}^{2} \cap X, \quad\left(u^{1}, v^{1}\right) \in \mathbb{L}^{2} .
$$


8 A transmission problem for beams on nonlinear supports

Suppose in addition that conditions (3.1)-(3.3) also hold. Then if $(u, v)$ is the solution of problem (1.1)-(1.6), one has the following decay rates:

(1) if $q>1$, then there exists a positive constant $C=C(E(0))$ such that

$$
E(t) \leq C(1+t)^{-2 /(q-1)}
$$

(2) if $q=1$, then there exist positive constants $C$ and $\mu$ such that

$$
E(t) \leq C E(0) e^{-\mu t}
$$

We will prove this theorem for strong solutions. Our conclusion follows by a standard density argument.

In order, we establish some auxiliary results related to the multipliers method. Let us introduce the functional

$$
R_{1}(t):=\int_{0}^{L_{0}} \rho_{1} u_{t} x u_{x} d x+\int_{L_{0}}^{L} \rho_{2} v_{t} x v_{x} d x
$$

In the following lemma we retrieve a part of the energy.

LEMMA 3.2. There exists a positive constant $C_{1}=C_{1}(E(0))$ such that

$$
\begin{aligned}
\frac{d}{d t} R_{1}(t) \leq & \frac{\rho_{2} L}{2}\left|v_{t}(L, t)\right|+C_{1}\left\{f(v(L, t)) v(L, t)+\left|g\left(v_{t}(L, t)\right)\right|\right\} \\
& -\frac{1}{2} \int_{0}^{L_{0}} \rho_{1}\left|u_{t}\right|+\beta_{1}\left|u_{x x}\right|-\frac{1}{2} \int_{L_{0}}^{L} \rho_{2}\left|v_{t}\right|+\beta_{2}\left|v_{x x}\right| d x
\end{aligned}
$$

for any strong solution of (1.1)-(1.6).

Proof. Multiplying (1.1) by $x u_{x},(1.2)$ by $x v_{x}$, integrating by parts, and using the boundary conditions (1.4)-(1.5) and (1.3), we arrive at the following identity:

$$
\begin{aligned}
\frac{d}{d t} R_{1}(t)= & \frac{L_{0}}{2}\left(\rho_{1}-\rho_{2}\right)\left|u_{t}\left(L_{0}, t\right)\right|^{2}+\frac{L_{0}}{2} \frac{\beta_{1}}{\beta_{2}}\left(\beta_{2}-\beta_{1}\right)\left|u_{x x}\left(L_{0}, t\right)\right|^{2} \\
& +\frac{\rho_{2} L}{2}\left|v_{t}(L, t)\right|^{2}-L\left(f(v(L, t))+g\left(v_{t}(L, t)\right)\right) v_{x}(L, t) \\
& -\frac{1}{2} \int_{0}^{L_{0}} \rho_{1}\left|u_{t}\right|^{2}+3 \beta_{1}\left|u_{x x}\right|^{2} d x-\frac{1}{2} \int_{L_{0}}^{L} \rho_{2}\left|v_{t}\right|^{2}+3 \beta_{2}\left|v_{x x}\right|^{2} d x .
\end{aligned}
$$

In view of the inequalities (3.2), the above equation reduces to

$$
\begin{aligned}
\frac{d}{d t} R_{1}(t) \leq & \frac{\rho_{2} L}{2}\left|v_{t}(L, t)\right|^{2} \underbrace{-L\left(f(v(L, t))+g\left(v_{t}(L, t)\right)\right) v_{x}(L, t)}_{:=I_{1}} \\
& -\frac{1}{2} \int_{0}^{L_{0}} \rho_{1}\left|u_{t}\right|^{2}+3 \beta_{1}\left|u_{x x}\right|^{2} d x-\frac{1}{2} \int_{L_{0}}^{L} \rho_{2}\left|v_{t}\right|^{2}+3 \beta_{2}\left|v_{x x}\right|^{2} d x .
\end{aligned}
$$


Now we will estimate $I_{1}$. Lemma 2.1 implies that $|v(L, t)| \leq C E^{1 / 2}(0)$ for some $C>0$, thus, as $f \in C^{1}(\mathbb{R})$ we have that $|f(v(L, t))| \leq C|v(L, t)|$ for some other positive constant $C=$ $C\left(E^{1 / 2}(0)\right)$. Applying Young's inequality and taking into account the preceding estimates, we get for $\eta>0$,

$$
\begin{aligned}
I_{1} & \leq \eta\left|v_{x}(L, t)\right|^{2}+C_{\eta}\left\{|f(v(L, t))|^{2}+\left|g\left(v_{t}(L, t)\right)\right|^{2}\right\} \\
& \leq \eta\left|v_{x}(L, t)\right|^{2}+C_{\eta}\left\{f(v(L, t)) v(L, t)+\left|g\left(v_{t}(L, t)\right)\right|^{2}\right\},
\end{aligned}
$$

from where by Lemma 2.1 follows that

$$
\begin{aligned}
I_{1} \leq & \eta C\left\{\int_{0}^{L_{0}} \beta_{1}\left|u_{x x}\right|^{2} d x+\int_{L_{0}}^{L} \beta_{2}\left|v_{x x}\right|^{2} d x\right\} \\
& +C_{\eta}\left\{f(v(L, t)) v(L, t)+\left|g\left(v_{t}(L, t)\right)\right|^{2}\right\} .
\end{aligned}
$$

Substitution of this inequality into (3.10) and fixing $\eta>0$ small our conclusion follows.

Our next step is to retrieve the remainder part of the energy. Let $(\varphi, \psi)$ be the solution of the stationary problem

$$
\begin{array}{ll}
\beta_{1} \varphi_{x x x x}=0 & \text { on }] 0, L_{0}\left[\times \mathbb{R}^{+},\right. \\
\beta_{2} \psi_{x x x x}=0 & \text { on }] L_{0}, L\left[\times \mathbb{R}^{+},\right.
\end{array}
$$

satisfying the boundary conditions

$$
\begin{gathered}
\varphi(0, t)=\varphi_{x}(0, t)=0, \\
\psi_{x x}(L, t)=0, \quad \psi(L, t)=v(L, t), \\
\varphi\left(L_{0}, t\right)-\psi\left(L_{0}, t\right)=0, \\
\varphi_{x}\left(L_{0}, t\right)-\psi_{x}\left(L_{0}, t\right)=0, \\
\beta_{1} \varphi_{x x}\left(L_{0}, t\right)-\beta_{2} \psi_{x x}\left(L_{0}, t\right)=0, \\
\beta_{1} \varphi_{x x x}\left(L_{0}, t\right)-\beta_{2} \psi_{x x x}\left(L_{0}, t\right)=0,
\end{gathered}
$$

which depend clearly on $v(L, t)$. We consider the following functional:

$$
R_{2}(t):=\int_{0}^{L_{0}} \rho_{1} u_{t} \varphi d x+\int_{L_{0}}^{L} \rho_{2} v_{t} \psi d x .
$$

Lemma 3.3. Given $\epsilon>0$, there exists a positive constant $C_{\epsilon}$ such that

$$
\begin{aligned}
\frac{d}{d t} R_{2}(t) \leq & \epsilon\left\{\int_{0}^{L_{0}} \rho_{1}\left|u_{t}\right|^{2}+\beta_{1}\left|u_{x x}\right|^{2} d x+\int_{L_{0}}^{L} \rho_{2}\left|v_{t}\right|^{2}+\beta_{2}\left|v_{x x}\right|^{2} d x\right\} \\
& +C_{\epsilon}\left\{\left|v_{t}(L, t)\right|^{2}+\left|g\left(v_{t}(L, t)\right)\right|^{2}\right\}-\frac{1}{2} f(v(L, t)) v(L, t)
\end{aligned}
$$

for any strong solution of (1.1)-(1.6). 
Proof. Multiplying (1.1) by $\varphi,(1.2)$ by $\psi$, integrating by parts and using boundary conditions (1.3)-(1.5) and (3.15), we have the following identity:

$$
\begin{aligned}
\frac{d}{d t} R_{2}(t)= & \int_{0}^{L_{0}} \rho_{1} u_{t} \varphi_{t} d x+\int_{L_{0}}^{L} \rho_{2} v_{t} \psi_{t} d x-\int_{0}^{L_{0}} \beta_{1} u_{x x} \varphi_{x x} d x \\
& -\int_{L_{0}}^{L} \beta_{2} v_{x x} \psi_{x x} d x-\left(f(v(L, t))+g\left(v_{t}(L, t)\right)\right) v(L, t) .
\end{aligned}
$$

On the other hand, multiplying (3.13) by $u-\varphi$, (3.14) by $v-\psi$, integrating by parts and using boundary conditions (1.3)-(1.5) and (3.15), we obtain

$$
\int_{0}^{L_{0}} \beta_{1} u_{x x} \varphi_{x x} d x+\int_{L_{0}}^{L} \beta_{2} v_{x x} \psi_{x x} d x=\int_{0}^{L_{0}} \beta_{1}\left|\varphi_{x x}\right|^{2} d x+\int_{L_{0}}^{L} \beta_{2}\left|\psi_{x x}\right|^{2} d x
$$

Since the right-hand side of this equality is positive, by substitution of this into (3.18) we arrive at

$$
\frac{d}{d t} R_{2}(t) \leq \int_{0}^{L_{0}} \rho_{1} u_{t} \varphi_{t} d x+\int_{L_{0}}^{L} \rho_{2} v_{t} \psi_{t} d x-f(v(L, t)) v(L, t)-g\left(v_{t}(L, t)\right) v(L, t) .
$$

Now, we will estimate the last term of the above inequality. Using Young's inequality and Lemma 2.1, we have for $\eta>0$,

$$
\begin{aligned}
\left|g\left(v_{t}(L, t)\right) v(L, t)\right| & \leq \eta|v(L, t)|^{2}+C_{\eta}\left|g\left(v_{t}(L, t)\right)\right|^{2} \\
& \leq \eta C\left\{\int_{0}^{L_{0}} \beta_{1}\left|u_{x x}\right|^{2} d x+\int_{L_{0}}^{L} \beta_{2}\left|v_{x x}\right|^{2} d x\right\}+C_{\eta}\left|g\left(v_{t}(L, t)\right)\right|^{2} .
\end{aligned}
$$

On the other hand, from the elliptic regularity of the system (3.13)-(3.15) there exists a constant $C>0$ such that

$$
\int_{0}^{L_{0}}|\varphi|^{2} d x+\int_{L_{0}}^{L}|\psi|^{2} d x \leq C|v(L, t)|^{2},
$$

and since the system (3.13)-(3.15) is linear we also have

$$
\int_{0}^{L_{0}}\left|\varphi_{t}\right|^{2} d x+\int_{L_{0}}^{L}\left|\psi_{t}\right|^{2} d x \leq C\left|v_{t}(L, t)\right|^{2}
$$

Applying Young's inequality to the two first terms of the right-hand side of (3.20) and using the above estimate we have for $\eta>0$,

$$
\begin{gathered}
\int_{0}^{L_{0}} \rho_{1} u_{t} \varphi_{t} d x \leq \eta \int_{0}^{L_{0}} \rho_{1}\left|u_{t}\right|^{2} d x+C_{\eta}\left|v_{t}(L, t)\right|^{2}, \\
\int_{L_{0}}^{L} \rho_{2} v_{t} \psi_{t} d x \leq \eta \int_{L_{0}}^{L} \rho_{2}\left|v_{t}\right|^{2} d x+C_{\eta}\left|v_{t}(L, t)\right|^{2} .
\end{gathered}
$$


By substitution of the estimates (3.21)-(3.24) into (3.20) and taking $\epsilon=\max \{\eta, \eta C\}$ we arrive at the desired result. This completes the proof of the lemma.

Now, we will summarize the results of the previous lemmas. Let us consider the following functional:

$$
R(t):=R_{1}(t)+2\left(C_{1}+1\right) R_{2}(t)
$$

where $C_{1}$ is the constant considered in Lemma 3.2.

Lemma 3.4. There exists a positive constant $C$ such that

$$
\frac{d}{d t} R(t) \leq-\frac{1}{2} E(t)+C\left\{g\left(v_{t}(L, t)\right) v_{t}(L, t)+\left[g\left(v_{t}(L, t)\right) v_{t}(L, t)\right]^{2 /(q+1)}\right\}
$$

for any strong solution of (1.1)-(1.6).

Proof. First, let $\epsilon_{0}$ be the solution of

$$
2\left(C_{1}+1\right) \epsilon_{0}=\frac{1}{4}
$$

Combining Lemmas 3.2 and 3.3 with $\epsilon=\epsilon_{0}$ and using the superlinearity of the function $f($ see $(3.1))$ we arrive at

$$
\frac{d}{d t} R(t) \leq-\frac{1}{2} E(t)+C\left\{\left|v_{t}(L, t)\right|^{2}+\left|g\left(v_{t}(L, t)\right)\right|^{2}\right\}
$$

Now, we will estimate the second term of the right-hand side of (3.28). From the hypothesis (3.3) we have the following estimates:

$$
\begin{aligned}
& \left|v_{t}(L, t)\right| \geq 1 \text { then }\left|v_{t}(L, t)\right|^{2}+\left|g\left(v_{t}(L, t)\right)\right|^{2} \leq C g\left(v_{t}(L, t)\right) v_{t}(L, t), \\
& \left|v_{t}(L, t)\right| \leq 1 \text { then }\left|v_{t}(L, t)\right|^{2}+\left|g\left(v_{t}(L, t)\right)\right|^{2} \leq C\left[g\left(v_{t}(L, t)\right) v_{t}(L, t)\right]^{2 /(q+1)} .
\end{aligned}
$$

Therefore, for any value of $v_{t}(L, t)$, we conclude that

$$
\left|v_{t}(L, t)\right|^{2}+\left|g\left(v_{t}(L, t)\right)\right|^{2} \leq C\left\{g\left(v_{t}(L, t)\right) v_{t}(L, t)+\left[g\left(v_{t}(L, t)\right) v_{t}(L, t)\right]^{2 /(q+1)}\right\} .
$$

In view of (3.28) the proof is complete.

Proof of Theorem 3.1. Now Lemma 3.4 plays an essential role. To prove the polynomial decay of the energy, we assume that $q>1$. Using Young's inequality is not difficult to show that there exists a positive constant $C$ such that

$$
|R(t)| \leq C E(t)
$$

Let us denote $\sigma:=(q-1) / 2$. Since

$$
\frac{d}{d t} E(t)=-g\left(v_{t}(L, t)\right) v_{t}(L, t)
$$


12 A transmission problem for beams on nonlinear supports

we get from estimate (3.31) that

$$
\begin{aligned}
\frac{d}{d t}\left[E^{\sigma} R\right](t) & \leq \sigma R(t) E^{\sigma-1}(t) \frac{d}{d t} E(t)+E^{\sigma}(t) \frac{d}{d t} R(t) \\
& \leq C E^{\sigma}(t) g\left(v_{t}(L, t)\right) v_{t}(L, t)+E^{\sigma}(t) \frac{d}{d t} R(t)
\end{aligned}
$$

Using Lemma 3.4 and estimate $E(t) \leq E(0)$, the above inequality can be written as

$$
\begin{aligned}
\frac{d}{d t}\left[E^{\sigma} R\right](t) \leq & -\frac{1}{2} E^{\sigma+1}(t)+C E^{\sigma}(0) g\left(v_{t}(L, t)\right) v_{t}(L, t) \\
& +C E^{(q+1) / 2}(0) E^{(q-1) /(q+1)}(t)\left[g\left(v_{t}(L, t)\right) v_{t}(L, t)\right]^{2 /(q+1)} .
\end{aligned}
$$

Using Young's inequality, the last term of the above inequality can be estimated by

$$
\begin{gathered}
C E^{(q+1) / 2}(0) E^{(q-1) /(q+1)}(t)\left[g\left(v_{t}(L, t)\right) v_{t}(L, t)\right]^{2 /(q+1)} \\
\leq \eta E^{\sigma+1}(t)+C_{\eta} E^{(q+1)^{2} / 4}(0) g\left(v_{t}(L, t)\right) v_{t}(L, t) .
\end{gathered}
$$

Taking $\eta=1 / 4$, inequality (3.34) becomes

$$
\frac{d}{d t}\left[E^{\sigma} R\right](t) \leq-\frac{1}{4} E^{\sigma+1}(t)+C g\left(v_{t}(L, t)\right) v_{t}(L, t),
$$

where $C$ is a constant which depends continuously on $E(0)$. Now, let us define the Lyapunov functional

$$
F(t):=N E(t)+\left[E^{\sigma} R\right](t) .
$$

Combining identity (3.32) with inequality (3.36) and taking $N$ large, we get

$$
\frac{d}{d t} F(t) \leq-\frac{1}{4} E^{\sigma+1}(t)
$$

On the other hand, in view of (3.31) we have that for $N$ large,

$$
\frac{N}{2} E(t) \leq F(t) \leq 2 N E(t)
$$

These two last inequalities imply that

$$
\frac{d}{d t} F(t) \leq-\alpha F^{\sigma+1}(t), \quad \alpha=(2 N)^{-(\sigma+1)}
$$

from where follows that

$$
F(t) \leq \frac{1}{\left(F^{-\sigma}(0)+\alpha \sigma t\right)^{1 / \sigma}} .
$$

Finally, the equivalence relation (3.39) implies the polynomial decay of the energy $E$. This proves the first part of Theorem 3.1. 
It remains to prove the exponential decay of the energy. To this end, we assume that $q=1$. From identity (3.32) and inequality (3.36) we have that the Lyapunov functional

$$
F(t):=N E(t)+R(t)
$$

satisfies

$$
\frac{d}{d t} F(t) \leq-\frac{1}{2} E(t)
$$

from where in view of (3.39) follows that for $N$ large,

$$
\frac{d}{d t} F(t) \leq-\frac{1}{4 N} F(t) \Longrightarrow F(t) \leq F(0) e^{-t / 4 N} .
$$

Finally, using equivalence relation (3.39) we have the exponential decay of the energy $E$. This completes the proof of Theorem 3.1.

Remarks 3.5. When considering 2-dimensional plates instead of 1-dimensional beams, there are mainly two kinds of difficulties. Firstly, the control of some unwanted tangential derivatives on the boundary where the support $f$ is acting. However, it seems that a compacity argument similar to the one in [14] may be used to show exponential decay for $q=1$. But polynomial decay for $q>1$ seems to be a harder question. The second kind of difficulties lies in the lack of formal results on the existence and regularity for stationary plate equations with transmission conditions similar to (1.3), which is essential when using multipliers techniques.

\section{Acknowledgment}

This work was partially supported by CNPq/Brazil.

\section{References}

[1] M. M. Cavalcanti, V. N. Domingos Cavalcanti, and T. F. Ma, Exponential decay of the viscoelastic Euler-Bernoulli equation with a nonlocal dissipation in general domains, Differential and Integral Equations 17 (2004), no. 5-6, 495-510.

[2] E. Feireisl, Nonzero time periodic solutions to an equation of Petrovsky type with nonlinear boundary conditions: slow oscillations of beams on elastic bearings, Annali della Scuola Normale Superiore di Pisa. Classe di Scienze 20 (1993), no. 1, 133-146.

[3] M. R. Grossinho and T. F. Ma, Symmetric equilibria for a beam with a nonlinear elastic foundation, Portugaliae Mathematica 51 (1994), no. 3, 375-393.

[4] B. V. Kapitonov and G. Perla Menzala, Energy decay and a transmission problem in electromagneto-elasticity, Advances in Differential Equations 7 (2002), no. 7, 819-846.

[5] __ Uniform stabilization and exact control of a multilayered piezoelectric body, Portugaliae Mathematica 60 (2003), no. 4, 411-454.

[6] J. E. Lagnese, Boundary Stabilization of Thin Plates, SIAM Studies in Applied Mathematics, vol. 10, SIAM, Pennsylvania, 1989.

[7] J. E. Lagnese and G. Leugering, Uniform stabilization of a nonlinear beam by nonlinear boundary feedback, Journal of Differential Equations 91 (1991), no. 2, 355-388.

[8] J.-L. Lions, Contrôlabilité Exacte, Perturbations et Stabilisation de Systèmes Distribués. Tome 1, Collection: Recherches en Mathématiques Appliquées, vol. 8, Masson, Paris, 1988. 


\section{A transmission problem for beams on nonlinear supports}

[9] J.-L. Lions and E. Magenes, Problèmes aux Limites non Homogènes et Applications. Vol. 1, Travaux et Recherches Mathématiques, no. 17, Dunod, Paris, 1968.

[10] W. Liu and G. Williams, The exponential stability of the problem of transmission of the wave equation, Bulletin of the Australian Mathematical Society 57 (1998), no. 2, 305-327.

[11] T. F. Ma, Existence results for a model of nonlinear beam on elastic bearings, Applied Mathematics Letters 13 (2000), no. 5, 11-15.

[12] B__ Boundary stabilization for a non-linear beam on elastic bearings, Mathematical Methods in the Applied Sciences 24 (2001), no. 8, 583-594.

[13] R. Ma and Y. An, Uniqueness of positive solutions of a class of ODE with nonlinear boundary conditions, Boundary Value Problems 2005 (2005), no. 3, 289-298.

[14] J. E. Muñoz Rivera and H. Portillo Oquendo, Transmission problem for viscoelastic beams, Advances in Mathematical Sciences and Applications 12 (2002), no. 1, 1-20.

[15] S. Nicaise, Boundary exact controllability of interface problems with singularities. II. Addition of internal controls, SIAM Journal on Control and Optimization 35 (1997), no. 2, 585-603.

[16] A. F. Pazoto and G. Perla Menzala, Uniform stabilization of a nonlinear beam model with thermal effects and nonlinear boundary dissipation, Funkcialaj Ekvacioj 43 (2000), no. 2, 339-360.

To Fu Ma: Department of Mathematics, State University of Maringá, 87020-900 Maringá, PR, Brazil

E-mail address: matofu@icmc.usp.br

Higidio Portillo Oquendo: Department of Mathematics, Federal University of Paraná, 81531-990 Curitiba, PR, Brazil

E-mail address: higidio@mat.ufpr.br 